\title{
IS THERE ALWAYS A UNIQUE EXTREMAL TEICHMÜLLER MAPPING?
}

\author{
KURT STREBEL
}

\begin{abstract}
A boundary correspondence of the circumference of the unit disk, which can be continued quasiconformally to the disk and which has the following property, is constructed: If there exists a Teichmüller mapping which is associated to a holomorphic quadratic differential and which is extremal for the boundary correspondence, then there is more than one such mapping.
\end{abstract}

Let there be given a boundary homeomorphism $h$ of the unit disk $D$ which is induced by a quasiconformal self-mapping $f$ of $D: h=f \mid \partial D$. The maximal dilatation of $f$ is denoted by $K$. The set of all quasiconformal self-mappings of $D$ with boundary values $h$ and with a fixed bound for the maximal dilatation is compact. Therefore there always exists a mapping $f_{0}$ in the class with smallest maximal dilatation $K_{0}$. It is called an extremal qc mapping for the given boundary homeomorphism $h$. If the dilatation $K$ of every mapping $f \neq f_{0}$ in the class is larger than $K_{0}, f_{0}$ is called uniquely extremal.

In the theory of extremal quasiconformal mappings, Teichmüller mappings play an important role. A quasiconformal mapping $w=f(z)$ is called a Teichmüller mapping if its complex dilatation $\kappa=f_{\bar{z}} / f_{z}$ is of the form $\kappa=k \bar{\varphi} /|\varphi|, 0 \leqslant k<1$, where $\varphi(z)$ is a meromorphic quadratic differential, i.e. a meromorphic function which transforms like the square of a derivative under a conformal change of the variable $z$. If $k$ is positive, the quadratic differential $\varphi$ is determined by $f$ up to a positive constant factor. Moreover, it also determines a quadratic differential $\psi(w)$, and if one chooses the factors and the constants of integration properly, $f$ has locally, except for isolated points, the representation

$$
f=\Psi^{-1} \circ F \circ \Phi \quad \text { with } \varphi=\Phi^{\prime 2}, \psi=\Psi^{\prime 2} .
$$

$F$ is the horizontal stretching by the factor $K=(1+k) /(1-k)$. Conversely, a mapping with the above representation is a Teichmüller mapping (for details see [1]).

There are boundary homeomorphisms $h$ which admit more than one extremal mapping. But the question, if every boundary homeomorphism $h$ admits, possibly among others, a Teichmüller extremal, is still open. On the other hand, the only uniquely extremal mappings known so far are Teichmüller mappings. In fact, every Teichmüller mapping associated with a quadratic differential $\varphi$ of finite norm

Received by the editors April 18, 1983.

1980 Mathematics Subject Classification. Primary 30C75; Secondary 30C60.

'This research was done during a stay at the University of Minnesota, Minneapolis. NSF Grant: MCS 77-28149 A01 and was presented in the Complex Analysis Seminar, University of Minnesota, Fall 1980. 
$\|\varphi\|=\iint|\varphi(z)| d x d y$ is uniquely extremal for its boundary values; but there are uniquely extremal (and not extremal) Teichmüller mappings with $\|\varphi\|=\infty$. So at this stage one still might expect a theorem of the form: Every boundary homeomorphism $h$ admits one and only one extremal Teichmüller mapping.

The following example shows that this expectation is wrong. We show the existence of a boundary homeomorphism $h$ which either does not admit a Teichmüller extremal or else admits many.

Let $R_{1}$ and $R_{2}$ be compact Riemann surfaces of genus $g \geqslant 2$ and let $f: R_{1} \rightarrow R_{2}$ be a quasiconformal mapping which is extremal in its homotopy class. We denote by $\hat{f}$ a lift of $f$ to the universal covering surfaces $\hat{R}_{1}$ and $\hat{R}_{2}$. If we represent $\hat{R}_{1}$ and $\hat{R}_{2}$ by unit disks $D_{1}:|z|<1$ and $D_{2}:|w|<1$, respectively, $\hat{f}$ becomes a quasiconformal mapping of $D_{1}$ onto $D_{2}$ of the same dilatation $K$ as $f$. It induces a boundary homeomorphism $h:|z|=1 \rightarrow|w|=1$. Let $\hat{K}_{0}$ be the maximal dilatation of an extremal qc mapping of $D_{1}$ onto $D_{2}$ with the boundary values $h$. Of course, $\hat{K}_{0} \leqslant K$. It is not yet known if $\hat{K}_{0}<K$ for all compact surfaces (except for the trivial case, where $f$ is conformal and, hence, $\hat{K}_{0}=K=1$ ), but there is at least an example of such a surface of genus 2 in [2]. We may therefore assume $R_{1}$ and $R_{2}$ have this property.

Suppose there exists an extremal Teichmüller mapping $\hat{f}_{0}$ for the boundary homeomorphism $h$. A lift $\hat{f}$ of any homeomorphism $f$ induces an isomorphism $\theta$ of the group $G_{1}$ of cover transformations $S_{1}$ of $\hat{R}_{1}$ onto the analogous group $G_{2}$ of $\hat{R}_{2}$. The mapping $\theta$ is defined by the relation $S_{1} \rightarrow S_{2}: \hat{f} \circ S_{1} \circ \hat{f}^{-1}=S_{2}$, where $S_{1}$ is a cover transformation of $\hat{R}_{1}$, and $S_{2}$ is its image. If the homeomorphisms $f$ and $g$ of $R_{1}$ onto $R_{2}$ are homotopic, they have homotopic lifts $\hat{f}$ and $\hat{g}$, which therefore induce the same isomorphism of $G_{1}$ onto $G_{2}$. Conversely, if the induced isomorphisms of two lifts $\hat{f}$ and $\hat{g}$ are the same, the traces $f$ and $g$ are homotopic [3].

Now let $z=S_{1}(\zeta)$ be a linear transformation of $D_{1}$ belonging to the group $G_{1}$ of cover transformations of $\hat{R}_{1}$, and let $S_{2}$ be its image by the isomorphism induced by $\hat{f}$. The mapping $\hat{f}$, and in particular the boundary homeomorphism $h=\hat{f} \mid \partial D_{1}$, satisfies the group relation $S_{2}^{-1} \circ h \circ S_{1}=h$. Therefore, $S_{2}^{-1} \circ \hat{f}_{0} \circ S_{1}$ has the same boundary values as $\hat{f}_{0}$, and of course the same dilatation $\hat{K}_{0}$. Moreover, it is a Teichmüller mapping. This follows at once from the second definition. In order to conclude it from the first one, we have to compute the complex dilatation. Let $d w=p(z) d z+q(z) d \bar{z}$ be the differential of $w=\hat{f}_{0}(z)$, and $z=S_{1}(\zeta)$. Then $d w=$ $p(z) S_{1}^{\prime}(\zeta) d \zeta+q(z) \overline{S_{1}^{\prime}}(\zeta) d \bar{\zeta}$, and, therefore,

$$
\kappa(z)=\frac{q(z)}{p(z)} \cdot \frac{\overline{S_{1}^{\prime}}(\zeta)}{S_{1}^{\prime}(\zeta)}=k \frac{\bar{\varphi}(z)}{|\varphi(z)|} \cdot \frac{\overline{S_{1}^{\prime 2}}(\zeta)}{\left|S_{1}^{\prime}(\zeta)\right|^{2}}=k \frac{\bar{\psi}(\zeta)}{|\psi(\zeta)|} .
$$

Here $\psi(\zeta)$ is the representation of the quadratic differential $\varphi$ in terms of the variable $\zeta$, because of the transformation law

$$
\psi(\zeta)=\varphi(z) S^{\prime}(\zeta)^{2}=\varphi(z)(d z / d \zeta)^{2} .
$$

If all the extremal Teichmüller mappings $S_{2}^{-1} \circ \hat{f}_{0} \circ S_{1}$ were the same, i.e, $\hat{f}_{0}$, it could be projected to $R_{1}, R_{2}$, and the projection $f_{0}$ would be homotopic to $f$. Therefore $\hat{K}_{0} \geqslant K$, which is a contradiction. 
Remark. Recently, E. Reich showed (Proc. Amer. Math. Soc. 88 (1983)) that for the horizontal stretching of the lying parabola there is another Teichmüller mapping with the same boundary values. The quadratic differential of this second mapping has a pole of the first order at the focus of the parabola, which is then mapped onto the focus of the image parabola. (It is not difficult to show that Reich's mapping of the punctured parabolas, i.e. considered as a mapping of the doubly connected domains, is uniquely extremal.)

Although not explicitly mentioned, the example constructed in this paper is meant to be a regular Teichmüller mapping (with holomorphic $\varphi$ ). For such mappings, both problems, the existence and the uniqueness, are still open. It is shown here that the answer cannot be positive to both of them.

\section{REFERENCES}

1. K. Strebel, Zur Frage der Eindeutigkeit extremaler quasikonformer Abbildungen des Einheitskreises. II, Comment. Math. Helv. 39 (1964), 77-89.

2. On lifts of extremal quasiconformal mappings, J. Analyse Math. 31 (1977), 191-203.

3. L. Bers, Quasiconformal mappings and Teichmüller's theorem, Analytic Functions (R. Nevanlinna et al., eds.), Princeton Univ. Press, Princeton, N. J., 1960, pp. 89-119.

4. O. Lehto, Group isomorphisms induced by quasiconformal mappings, Contributions to Analysis, Academic Press, New York, 1974, pp. 241-244.

Mathematisches InSTITUT, UNIVERSITÄt ZURICH, 80001 ZURICH, SWITZERLAND 